\title{
siRNA-mediated silencing of Wnt5a regulates inflammatory responses in atherosclerosis through the MAPK/NF-кB pathways
}

\author{
LEI YANG $^{1}$, YINGJIE CHU ${ }^{1}$, YUHANG WANG ${ }^{1}$, XIANGMEI ZHAO ${ }^{1}$, WENKE XU $^{1}$, PEIRONG ZHANG ${ }^{1}$, \\ XIAOYU LIU ${ }^{1}$, SHUJUAN DONG ${ }^{1}$, WENQI HE ${ }^{1}$ and CHUANYU GAO ${ }^{2}$ \\ Departments of ${ }^{1}$ Emergency Medicine, and ${ }^{2}$ Cardiology, Henan Provincial People's Hospital, \\ Zhengzhou, Henan 450003, P.R. China
}

Received March 17, 2014; Accepted July 8, 2014

DOI: $10.3892 /$ ijmm.2014.1860

\begin{abstract}
Previous studies have demonstrated that the aberrant expression of Wnt5a occurs in atherosclerotic lesions. However, the precise role of Wnt5a in the pathogenesis of atherosclerosis remains largely unknown. The present study was undertaken to determine whether the RNA interference of Wnt5a in vivo by adenovirus (Ad)-mediated small interfering RNA (siRNA) transfection is capable of inhibiting the progression of atherosclerosis. Recombinant adenovirus carrying siRNA targeting Wnt5a (Ad-Wnt5a siRNA) was designed. Male apolipoprotein E-deficient $\left(\mathrm{ApoE}^{-/-}\right)$mice were fed a high-fat diet to induce the pathogenesis of atherosclerosis. Mice were randomly divided into 3 groups $(n=15$ in each group): the mock group, which received treatment with phosphate-buffered saline (PBS); the Ad-NC group, which received treatment with Ad-non-specific siRNA; and the Ad-Wnt5a siRNA group, which received treatment with Ad-Wnt5a siRNA. Treatment with Ad-Wnt5a siRNA markedly inhibited the mRNA and protein expression of Wnt5a in the aortic tissues. The knockdown of Wnt5a had no significant effect on blood lipid levels, but it suppressed atherosclerotic development and increased plaque stability, which was determined by hematoxylin and eosin staining, picrosirius red staining and Oil Red O staining. Furthermore, the mRNA and protein expression of inflammatory cytokines, including
\end{abstract}

Correspondence to: Dr Yingjie Chu, Department of Emergency Medicine, Henan Provincial People's Hospital, No. 7 Weiwu Road, Zhengzhou, Henan 450003, P.R. China

E-mail: leilei1747@126.com

Abbreviations: ApoE ${ }^{-/}$, apolipoprotein E-deficient; COX-2, cyclooxygenase-2; JNK, Jun N-terminal kinase; MAPK, mitogen-activated protein kinase; MCP-1, monocyte chemotactic protein-1; MMP-2, matrix metalloproteinase-2; MMP-9, matrix metalloproteinase-9; NF- $\kappa B$, nuclear factor- $\kappa B$; PBS, phosphate-buffered saline; siRNA, small interfering RNA; TC, total cholesterol; TG, triglyceride; HDL, high-density lipoprotein; LDL, low-density lipoprotein.

Key words: atherosclerosis, Wnt5a, inflammation, small interfering RNA monocyte chemotactic protein-1 (MCP-1), cyclooxygenase-2 (COX-2), matrix metalloproteinase (MMP)-2 and MMP-9 was significantly downregulated in the Ad-Wnt5a siRNA group. In addition, the knockdown of Wnt5a inhibited the nuclear factor- $\kappa \mathrm{B}(\mathrm{NF}-\kappa \mathrm{B})$ and mitogen-activated protein kinase (MAPK) signaling pathways. These results demonstrate that Ad-mediated Wnt5a silencing in vivo attenuates the development of atherosclerotic disease by reducing inflammatory mediators involved in the MAPK/NF- $\mathrm{BB}$ pathways.

\section{Introduction}

Atherosclerosis, a chronic inflammatory disease, is a major factor leading to cardiovascular complications (1). Following the upregulation of inflammatory chemokines, monocytes adhere to endothelial cells in the arterial wall and differentiate into macrophages, which ingest lipoproteins and form foam cells. Vascular smooth muscle cells proliferate and synthesize extracellular matrix proteins. The above processes lead to plaque formation (2).

Wnt5a is a highly conserved secreted glycoprotein of the Wnt protein family, which activates the $\beta$-catenin-independent signaling pathway, including the planar cell polarity (PCP) pathway and the $\mathrm{Ca}^{2+}$ pathway, which then further activates downstream signals, including Jun N-terminal kinase (JNK), protein kinase $\mathrm{C}(\mathrm{PKC})$ and $\mathrm{Ca}^{2+} /$ calmodulin-dependent kinase II (CaMKII) (3). Wnt5a has been implicated in inflammatory diseases, including rheumatoid arthritis, atherosclerosis, psoriasis and sepsis, suggesting that it plays a critical biological role in the regulation of inflammation (4-7). In vitro studies have demonstrated that Wnt5a promotes the expression of inflammatory cytokines, chemokines and matrix metalloproteinases (MMPs) (8). Wnt5a can activate nuclear factor- $\kappa \mathrm{B}$ $(\mathrm{NF}-\kappa \mathrm{B})$ which is involved in the expression of inflammatory genes (9). Wnt5a also stimulates chemotactic migration and chemokine production by activating the p38 and extracellular signal-regulated kinase (ERK) pathways (10).

Christman et al (5) reported that Wnt5a expression was expressed in human and murine atherosclerotic lesions. It has recently been demonstrated that Wnt5a levels are elevated in the serum of atherosclerotic patients relative to healthy controls and that the expression of Wnt5a is increased in advanced 
human atherosclerotic lesions (11). These data suggest an active role of Wnt5a in the pathogenesis of atherosclerosis. However, the direct effects of Wnt5a on atherosclerotic lesions and the underlying mechanisms have not been well delineated.

In the present study, we used adenovirus (Ad)-mediated small interfering RNA (siRNA) to target Wnt5a in a mouse model of atherosclerosis. Subsequently, the effects of Wnt5a knockdown on atherosclerotic lesions and the potential mechanisms involved were investigated.

\section{Materials and methods}

Animal model. Eight-week-old male apolipoprotein E-deficient $\left(\mathrm{ApoE}^{-/}\right)$mice with a $\mathrm{C} 57 \mathrm{BL} / 6 \mathrm{~J}$ genetic background were obtained from Beijing University of Medicine Laboratory (Beijing, China). All experimental protocols were approved by the Institutional Animal Care Committee of Henan Provincial People's Hospital, Zhengzhou, China. All the mice were kept at $25^{\circ} \mathrm{C}$ on a 12 -h light/dark cycle and fed a high-fat diet ( $15 \%$ fat, $0.25 \%$ cholesterol). All efforts were made to minimize suffering. Mice were euthanized by an injection with pentobarbital sodium $(100 \mathrm{mg} / \mathrm{kg})$ intraperitoneally.

Construction of recombinant adenovirus carrying siRNA. According to the Wnt5a gene sequence (GenBank accession no. NM_009524), an effective siRNA for Wnt5a (sense, 5'-GAAGCCCAUUGGAAUAUUATT-3' and antisense, 5'-UAAUAUUCCAAUGGGCUUCTT-3') was designed and synthesized by Genepharma Co., Ltd. (Shanghai, China). Non-specific siRNA sequences (sense, 3'-UUCUCCGAACG UGUCACGUUU-5' and antisense, 3'-ACGUGACACGUUC GGAGAAUU-5') were used as the control. The recombinant adenovirus was constructed as previously described (12). The siRNA sequences were amplified and subcloned into the pAdTrack-cytomegalovirus (CMV) plasmid, an adenoviral shuttle plasmid. Subsequently, the recombinant shuttle plasmids, pAdTrack-CMV and pAdEasy-1, were homologously recombined in Escherichia coli strain BJ5183. The obtained recombinant plasmids were then transfected into human embryonic kidey (HEK)-293 cells (Type Culture Collection of the Chinese Academy of Sciences, Shanghai, China) to generate recombinant adenovirus. The virus was amplified and purified, and titers were determined using the p24 ELISA kit (Cell Biolabs, Inc., San Diego, CA, USA).

Treatment of mice with recombinant adenovirus. After 10 weeks on a high-fat diet, the mice were randomly divided into 3 groups $(n=15$ in each group): the mock group which received a $200-\mu 1$ phosphate-buffered saline (PBS) injection; the Ad-NC group which received a $200-\mu 1$ ( $1 \times 10^{10}$ plaque-forming units of virus) injection of recombinant adenovirus expressing non-specific siRNA; the Ad-Wnt5a siRNA group which received 200- $\mu$ l ( $1 \times 10^{10}$ plaque-forming units) injection of recombinant adenovirus expressing Wnt5a siRNA. Two weeks later, a second injection was administered in a similar fashion as described above, and the mice were euthanized for analysis 2 weeks after the second injection. Blood samples were collected by cardiac puncture from mice fasted overnight and concentrations of plasma total cholesterol (TC), triglyceride (TG), high-density lipoprotein (HDL) and low-density lipoprotein (LDL) were detected using the ELISA kit (Shanghai Bangyi Biotechnology Co., Ltd., Shanghai, China) on an automatic analyzer (Roche P800; Roche Diagnostics, Indianapolis, IN, USA).

Analysis of atherosclerosis. Prior to carotid artery isolation, the mouse hearts were perfused with PBS, followed by $4 \%$ paraformaldehyde for $30 \mathrm{~min}$ under physiological pressure. Afterwards, the isolated carotid artery was fixed with $4 \%$ paraformaldehyde for $12 \mathrm{~h}$, then embedded in paraffin and cut into $5-\mu \mathrm{m}$ serial sections. The paraffin sections were prepared and stained with hematoxylin and eosin (H\&E) (Sigma, St. Louis, MO, USA). For the detection of collagen, the sections were stained with picrosirius red (Sigma). Lipid-rich lesions were identified by Oil Red O (Sigma) staining. The plaque area, collagen area and lipid area were observed and calculated using Image-Pro Plus 6.0 software (Media Cybernetics Inc., Rockville, MD, USA).

Quantitative reverse transcription-polymerase chain reaction $(R T-q P C R)$. Total RNA was extracted from the carotid arteries using the RNeasy Fibrous Tissue mini kit (Qiagen, Valencia, CA, USA) and reverse transcribed into cDNA using a PrimeScript ${ }^{\mathrm{TM}}$ 1st Strand cDNA Synthesis kit (Takara, Dalian, China). Quantitative PCR was performed using SYBR ${ }^{\circledR}$ Premix DimerEraser ${ }^{\mathrm{TM}}$ (Takara) to detect the mRNA expression of Wnt5a, cyclooxygenase-2 (COX-2), MMP-2, MMP-9 and monocyte chemotactic protein-1 (MCP-1). The primer sequences used are listed in Table I. Quantitative measurements were determined using the $\Delta \Delta \mathrm{Ct}$ method and glyceraldehyde 3-phosphate dehydrogenase (GAPDH) was used as the internal standard for all samples. The RT-qPCR reactions were performed in triplicate.

Western blot analysis. Proteins from the carotid arteries were isolated using RIPA Lysis buffer (Beyotime, Haimen, China) containing $100 \mu \mathrm{g} / \mathrm{ml}$ phenylmethylsulfonyl fluoride (PMSF; Beyotime). Protein concentrations were determined using a BCA assay. Equal amounts of protein were separated by SDS-PAGE and then transferred onto nitrocellulose membranes (Bio-Rad, Hercules, CA, USA). The membranes were blocked in $5 \%$ skimmed milk for $1 \mathrm{~h}$ at room temperature and incubated with primary antibodies overnight at $4^{\circ} \mathrm{C}$. Subsequently, they were incubated with horseradish peroxidase (HRP)-conjugated secondary antibodies for $1 \mathrm{~h}$ at room temperature. The membranes were visualized using an Odyssey imaging system (LI-COR Biosciences, Lincoln, NE, USA). The following antibodies were used: rabbit anti-Wnt5a $(0.5 \mu \mathrm{g} / \mathrm{ml})$, rabbit antiMMP-2 (1:500), rabbit anti-MMP-9 (1:600), rabbit anti-COX-2 (1:600), rabbit anti-GAPDH $(0.5 \mu \mathrm{g} / \mathrm{ml})$, goat anti-rat IgG H\&L (HRP) (1:2,000), goat anti-rabbit IgG H\&L (HRP) $(1: 2,000)$ (all purchased from Abcam, Cambridge, MA), rabbit anti-MCP-1 (1:600), rabbit anti-phospho-specific Thr202/Tyr204-ERK1/2 (1:1,000), rabbit anti-phospho-SAPK/JNK Thr183/Tyr185 (1:1,000), rabbit antiphospho-p38 mitogen-activated protein kinase (MAPK) Thr180/Tyr182 (1:1,000) and rabbit antiphospho-NF-кB p65 Ser536 (1:1,000) (all purchased from Cell Signaling Technology (Danvers, MA, USA).

Statistical analysis. Values are expressed as the means \pm standard deviation (SD). Statistical significance of differences 
Table I. Primers used for RT-qPCR.

\begin{tabular}{lll}
\hline Gene & \multicolumn{1}{c}{ Forward primer $\left(5^{\prime} \rightarrow 3^{\prime}\right)$} & \multicolumn{1}{c}{ Reverse primer $\left(5^{\prime} \rightarrow 3^{\prime}\right)$} \\
\hline Wnt5a & AATCCACGCTAAGGGTTCCTATGAG & AGCCAGCACGTCTTGAGGCTA \\
COX-2 & CCCAGAGCTCCTTTTCAACC & ATTTGGCACATTTCTTCCCC \\
MMP-2 & ACCCAGATGTGGCCAACTAC & TACTTTTAAGGCCCGAGCAA \\
MMP-9 & ATGATGGAGGAGAAGCAGTC & AGGTGAAGGGAAAGTGACAT \\
MCP-1 & TTAAAAACCTGGATCGGAACCAA & GCATTAGCTTCAGATTTACGGG \\
GAPDH & AAGGGTCATCATCTCTGCCC & GTGATGGCATGGACTGTGGT
\end{tabular}

RT-qPCR, quantitative reverse transcription-polymerase chain reaction; COX-2, cyclooxygenase-2; MMP, matrix metalloproteinase; MCP-1, monocyte chemotactic protein-1; GAPDH, glyceraldehyde 3-phosphate dehydrogenase.
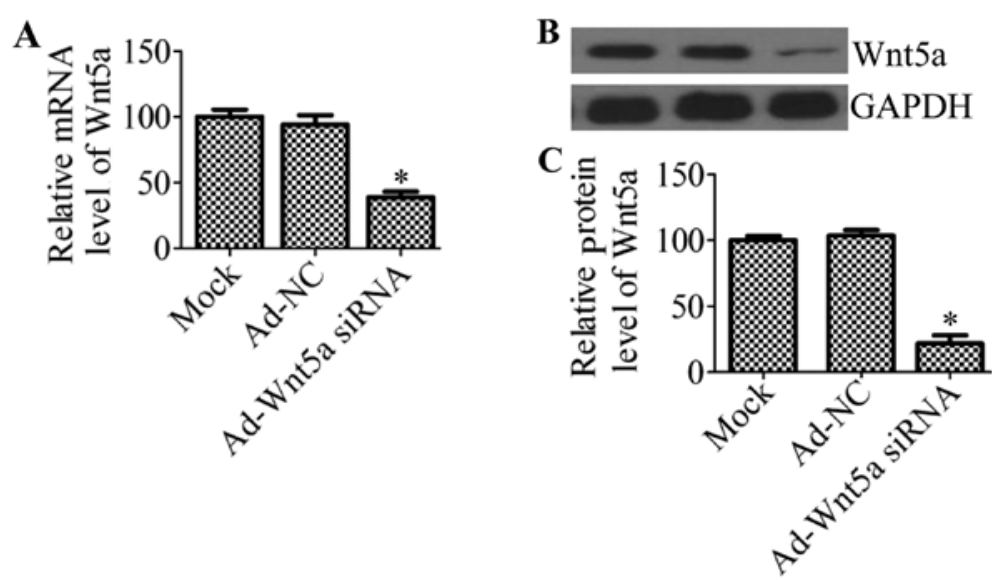

Figure 1. Effect of adenovirus-mediated small interfering RNA (siRNA) on the expression of Wnt5a in vivo. (A) RT-qPCR was performed to analyze the mRNA expression of Wnt5a in carotid artery tissues. (B) Western blot analysis was used for the detection of the protein expression of Wnt5a. Glyceraldehyde 3-phosphate dehydrogenase (GAPDH) was used as a loading control. (C) Protein expression was analyzed using Image-Pro Plus 6.0 software and normalized to GAPDH. Mice in the mock group were treated with phosphate-buffered saline (PBS); mice in the Ad-NC group were treated with Ad-mediated non-specific siRNA (Ad-NC); mice in the Ad-Wnt5a siRNA group were treated with Ad-mediated Wnt5a siRNA. Data are expressed as the means \pm standard deviation (SD). "P<0.05.

between two groups was determined using the Student's t-test, and that among multiple groups was determined by one-way ANOVA. A P-value of $<0.05$ was considered to indicate a statistically significant difference. All statistical analyses were performed using SPSS software version 11.5 (SPSS Inc., Chicago, IL, USA).

\section{Results}

Effective knockdown of Wnt5a by Ad-mediated siRNA in $A p o E^{-/-}$mice. After adenovirus carrying Wnt5a siRNA was administered to the mice in vivo, Wnt5a expression in the carotid artery tissues was determined by RT-qPCR and western blot analysis. The results revealed that the mRNA expression of Wnt5a was significantly decreased by $61.3 \%$ compared with that of the Ad-NC group and mock group $(\mathrm{P}<0.05)$ (Fig. 1A). These results were further confirmed by western blot analysis (Fig. 1B), which indicated that the protein expression of Wnt5a was also markedly decreased by $76.5 \%$ (Fig. 1C) in the mice treated with Ad-Wnt5a siRNA compared with the controls.

Ad-Wnt5a siRNA has no effect on plasma lipid levels. To determine the effects of Wnt5a knockdown on the mouse model of atherosclerosis, the serum lipid levels of the mice in the 3 groups were detected. There was no significant difference in the serum levels of TC, TG, HDL and LDL between the mock group and NC group, suggesting that treatment with Ad-NC had no effects on the serum lipid profiles of the $\mathrm{ApoE}^{-/-}$ mice (Fig. 2). Compared with the mock group or NC group, the administration of Ad-Wnt5a siRNA also had no significant effect on blood lipid levels.

Treatment with Ad-Wnt5a siRNA improves the stability of atherosclerotic plaque. In order to further investigate the effects of treatment with Ad-Wnt5a siRNA on atherosclerosis, we determined the atherosclerotic lesion areas by $\mathrm{H} \& \mathrm{E}$ staining. Compared with the mock group or Ad-NC group, the mice which were transfected with Ad-Wnt5a siRNA exhibited a decreased lesion area (Fig. 3A), suggesting that the knockdown of Wnt5a suppressed atherosclerotic development in the $\mathrm{ApoE}^{-/-}$mice. In order to assess whether the silencing of Wnt5a expression affects plaque stability, we analyzed the collagen content and lipid area in atherosclerotic plaque. Using picrosirius red staining, we found that siRNA targeting Wnt5a significantly increased the collagen area (Fig. 3B). Moreover, we found that treatment with Ad-Wnt5a siRNA decreased the lipid content in the plaque compared with the mock or NC group (Fig. 3C). 
A

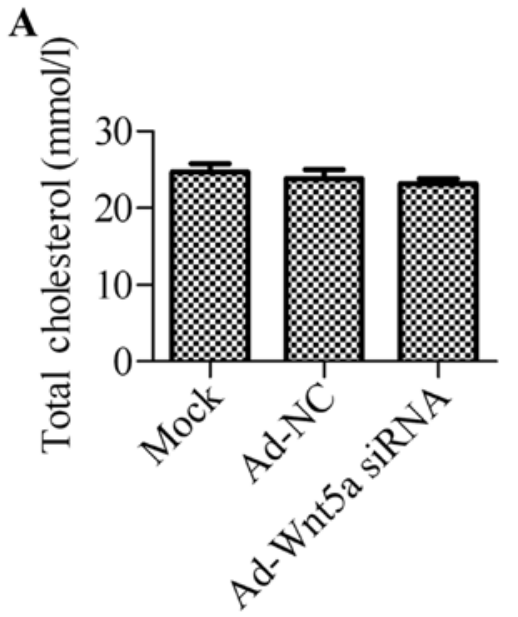

C

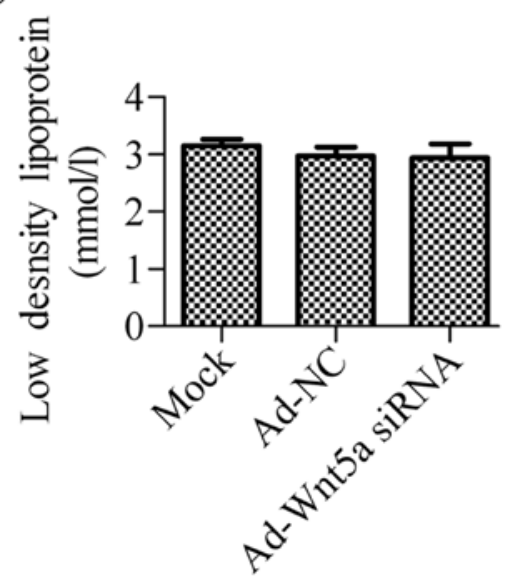

B

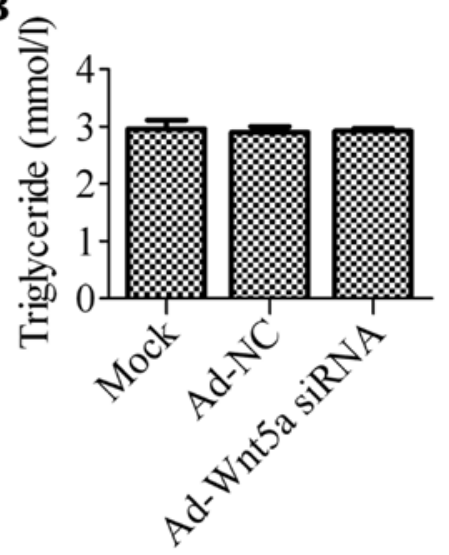

D

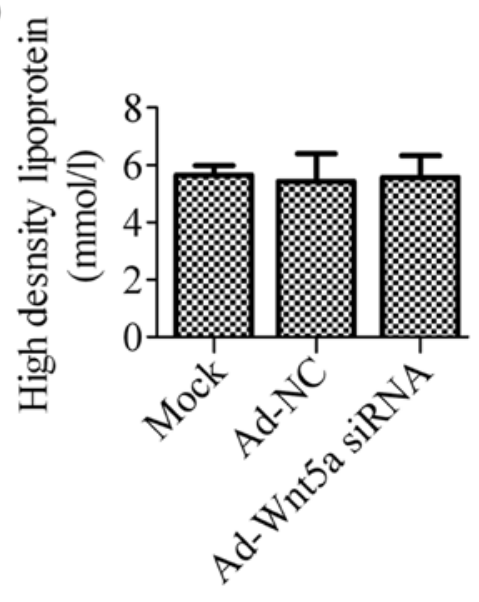

Figure 2. Effects of Ad-Wnt5a small interfering RNA (siRNA) on plasma lipid level. Plasma levels of (A) total cholesterol, (B) triglyceride, (C) low-density lipoprotein (LDL) cholesterol and (D) high-density lipoprotein (HDL) cholesterol in the 3 groups of mice. Data are expressed as the means \pm standard deviation (SD).
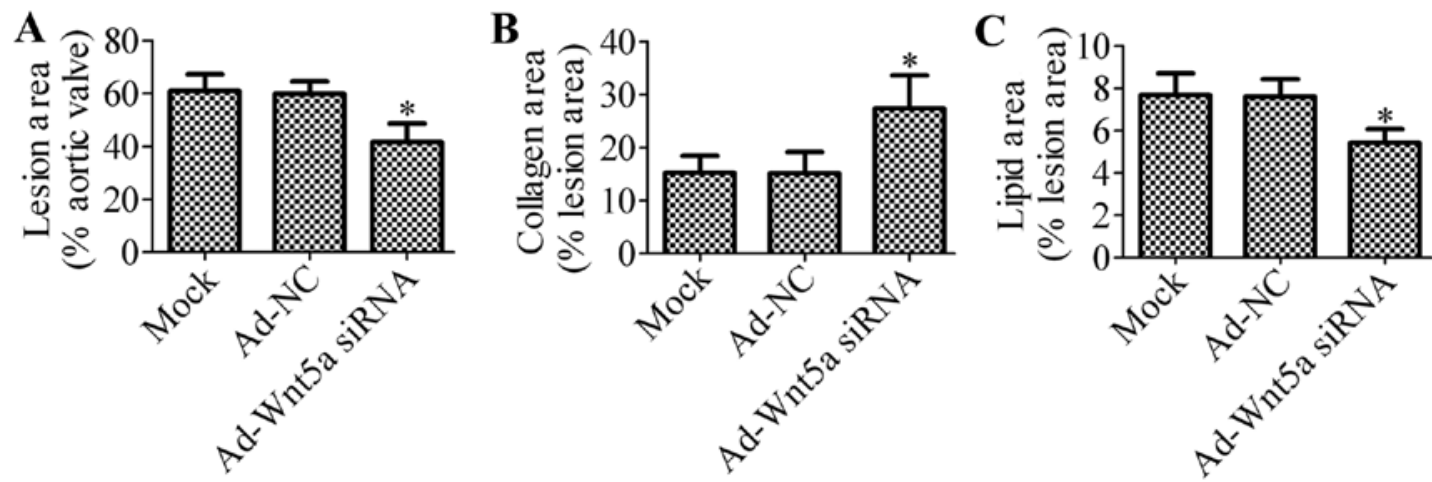

Figure 3. Effect of Ad-Wnt5a small interfering RNA (siRNA) on atherosclerotic lesion development and plaque stability. (A) Plaque area of the carotid artery was analyzed by hematoxylin and eosin (H\&E) staining. Plaque area was quantified as a percentage of the aortic valve. (B) Collagen content was analyzed by picrosirius red staining and (C) lipid content was analyzed by Oil Red O staining, which was quantified as a percentage of the total plaque area. Data are expressed as the means \pm standard deviation (SD). ${ }^{*} \mathrm{P}<0.05$.

Silencing of Wnt5a alters the expression of inflammatory mediators. Inflammatory factors have been reported to play an important role in atherosclerotic plaque formation and stability (13). Additionally, Wnt5a is involved in the regulation of inflammation (7). Thus, we then investigated whether the knockdown of Wnt5a has an effect on the inflammatory factors involved in atherosclerotic plaque formation and stability. The levels of inflammatory factors, such as MCP-1, COX-2, MMP-2 and MMP-9 were analyzed by RT-qPCR and western blot analysis. Compared with the mock and NC groups, Ad-Wnt5a siRNA significantly downregulated the mRNA expression of MCP-1, COX-2, MMP-2 and MMP-9 in the atherosclerotic lesions (Fig. 4A). Moreover, the protein expression of MCP-1, COX-2, MMP-2 and MMP-9 was also decreased by Ad-Wnt5a siRNA (Fig. 4B). These results indicate that the knockdown of Wnt5a inhibits the inflammatory response in atherosclerosis. 


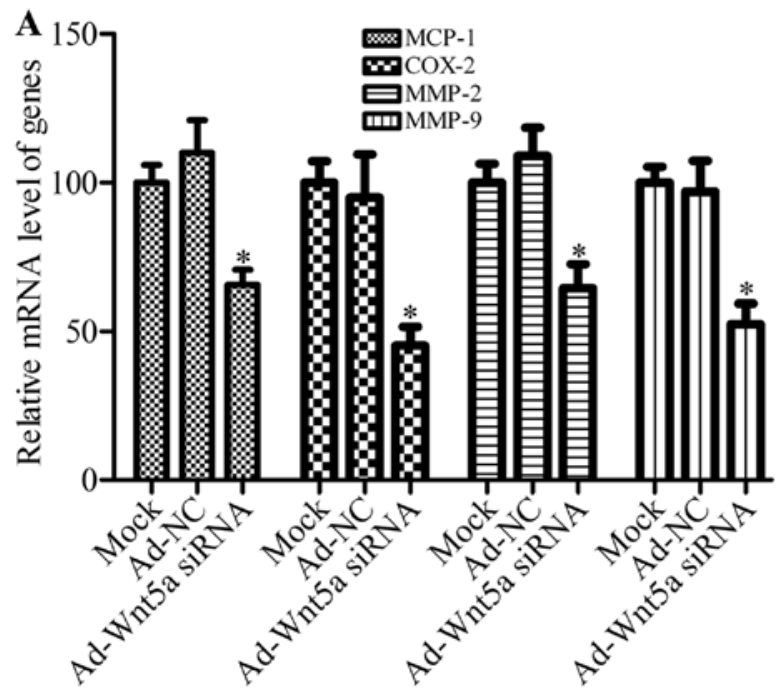

B

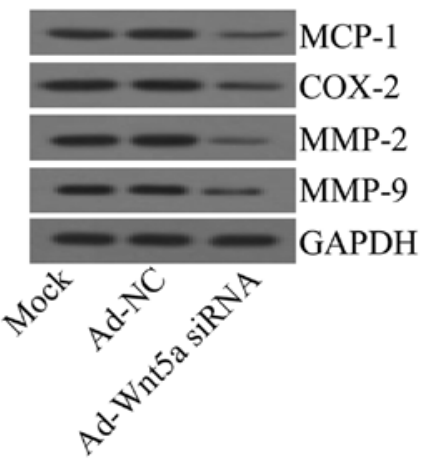

Figure 4. Effect of Ad-Wnt5a small interfering RNA (siRNA) on the mRNA and protein expression of inflammatory mediators, monocyte chemotactic protein-1 (MCP-1), cyclooxygenase-2 (COX-2), matrix metalloproteinase (MMP)-2 and MMP-9. Detection of (A) mRNA expression and (B) protein expression levels of genes by RT-qPCR and western blot analysis, respectively. Glyceraldehyde 3-phosphate dehydrogenase (GAPDH) was used as a control. " $\mathrm{P}<0.05$.

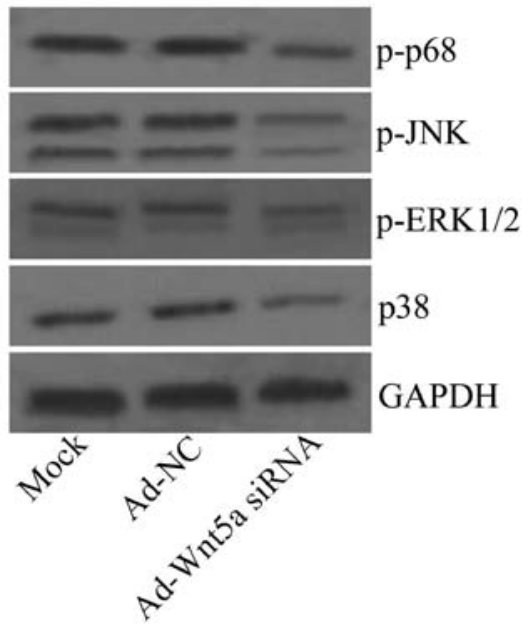

Figure 5. Effect of Ad-Wnt5a small interfering RNA (siRNA) on nuclear factor- $\mathrm{\kappa B}(\mathrm{NF}-\mathrm{\kappa} \mathrm{B})$ and mitogen-activated protein kinase (MAPK) signaling pathways. Western blot analysis of phosphorylated p65, Jun N-terminal kinase (JNK), extracellular signal-regulated kinase (ERK)1/2 and $\mathrm{p} 38$. Glyceraldehyde 3-phosphate dehydrogenase (GAPDH) was used as a control.

Ad-Wnt5a siRNA inhibits the activation of the $N F-\kappa B$ and $M A P K$ signaling pathways. The activation of the $\mathrm{NF}-\kappa \mathrm{B}$ and MAPK pathways is known to result in the production of inflammatory cytokines and chemokines $(14,15)$, which is constitutively activated in atherosclerosis. To investigate whether Ad-Wnt5a siRNA is capable of activating NF- $\mathrm{NB}$ and MAPK pathways, we examined the effects of treatment with Ad-Wnt5a siRNA on p65, JNK, ERK1/2 and p38 phosphorylation in by western blot analysis. The results revealed that Ad-Wnt5a siRNA attenuated p65, JNK, ERK1/2 and p38 phosphorylation compared with the mock and Ad-NC groups (Fig. 5), suggesting that the knockdown of Wnt5a inhibited the $\mathrm{NF}-\kappa \mathrm{B}$ and MAPK signaling pathways.

\section{Discussion}

In the present study, we found that the silencing of Wnt5a in vivo reduced the plaque area and increased plaque stability in an animal model of atherosclerosis. The potential mechanisms may involve the NF- $\kappa \mathrm{B}$ and MAPK pathways, through which Wnt5a downregulated inflammatory mediators. To the best of our knowledge, our study is the first to delineate the direct effects of Ad-Wnt5a siRNA on atherosclerotic lesions and the underlying mechanisms in $\mathrm{ApoE}^{-/-}$mice in vivo.

Atherosclerosis is a serious inflammatory disorder that is associated with the upregulation of inflammatory makers. Therefore, atherosclerotic development may be inhibited by reducing the levels of inflammatory mediators. COX-2 is a key regulator of inflammatory processes and is expressed in atherosclerotic lesions from humans and mice $(16,17)$. COX-2 has been shown to promote early atherosclerotic lesion formation in $\mathrm{ApoE}^{-/-}$mice (18). It has been demonstrated that Wnt5a induces the expression of COX-2 in endothelial cells (9). MCP-1 is a member of the chemokine family and plays an important role in the initiation of atherosclerosis. It has previously been demonstrated that the absence of MCP-1 exerts protective effects against macrophage recruitment and atherosclerotic lesion formation in apolipoprotein B (ApoB) transgenic mice (19). In the present study, we found that the silencing of Wnt5a downregulated the expression of COX-2 and MCP-1, and decreased the plaque area of the aortic root in $\mathrm{ApoE}^{-/-}$mice, suggesting that the silencing of Wnt5a attenuates atherosclerotic lesion formation by reducing the levels of COX-2 and MCP-1.

The majority of atherosclerotic plaques are stable, but a few become vulnerable and lead to myocardial infarction (MI) and stroke (20-22). Plaque rupture is a major type of vulnerable plaque (23). A previous study indicated that an increased collagen-to-lipid ratio is an important indicator of plaque rupture (24). MMP-2 and MMP-9, as mediators of extracellular cell matrix degradation, play important roles in plaque rupture 
by degrading collagen (25). In the current study, we found a reduced collagen-to-lipid ratio and a decreased expression of MMP-2 and MMP-9 in the Ad-Wnt5a siRNA group, suggesting that the silencing of Wnt5a enhances plaque stability.

In the progression of an atherosclerotic lesion, $\mathrm{NF}-\kappa \mathrm{B}$ activation promotes the expression of $\mathrm{COX}$, cytokines, chemokines and adhesion molecules (14). NF- $\kappa \mathrm{B}$ has also been reported to regulate metalloproteinase expression, particularly that of MMP-2 and MMP-9 (26). The inhibition of NF- $\kappa \mathrm{B}$ signaling attenuates the pathogenesis of atherosclerosis (27). It has previously been demonstrated that MAPK activation facilitates foam cell formation and MAPK inactivation is an interesting target for drug therapy to reduce atherosclerotic lesion formation (28). In this study, we measured NF- $\kappa \mathrm{B}$ activity and found that the silencing of Wnt5a decreased p65, JNK, ERK1/2 and p38 phosphorylation. This indicates that the silencing of Wnt5a may suppress NF- $\mathrm{BB}$ and MAPK activity, reducing the levels of COX-2, MCP-1, MMP-2 and MMP-9.

Taken together, our results demonstrate that the silencing of Wnt5a inhibits NF- $\mathrm{BB}$ and MAPK signaling, thereby modulating the level of inflammation in atherosclerotic conditions and protecting against vascular injury in atherosclerosis. The present study provides evidence that Wnt5a may be used as an effective molecular target for the gene therapy of atherosclerosis. However, further investigations are warranted to delineate the precise molecular mechanisms of Wnt5a in regulating the inflammatory process in atherosclerosis.

\section{Acknowledgements}

This study was supported by the State Key Clinical Specialty Construction Project and the Key Project of Science and Technology of Henan (grant no. 132102310080).

\section{References}

1. Glass CK and Witztum JL: Atherosclerosis. the road ahead. Cell 104: 503-516, 2001

2. Li AC and Glass CK: The macrophage foam cell as a target for therapeutic intervention. Nat Med 8: 1235-1242, 2002.

3. Kikuchi A, Yamamoto H, Sato A and Matsumoto S: Wnt5a: its signalling, functions and implication in diseases. Acta Physiol (Oxf) 204: 17-33, 2012.

4. Sen M, Lauterbach K, El-Gabalawy H, et al: Expression and function of wingless and frizzled homologs in rheumatoid arthritis. Proc Natl Acad Sci USA 97: 2791-2796, 2000.

5. Christman MA II, Goetz DJ, Dickerson E, et al: Wnt5a is expressed in murine and human atherosclerotic lesions. Am J Physiol Heart Circ Physiol 294: H2864-H2870, 2008.

6. Reischl J, Schwenke S, Beekman JM, et al: Increased expression of Wnt5a in psoriatic plaques. J Invest Dermatol 127: 163-169, 2007.

7. Pereira C, Schaer DJ, Bachli EB, Kurrer MO and Schoedon G: Wnt5A/CaMKII signaling contributes to the inflammatory response of macrophages and is a target for the antiinflammatory action of activated protein $\mathrm{C}$ and interleukin-10. Arterioscler Thromb Vasc Biol 28: 504-510, 2008.
8. Halleskog C, Dijksterhuis JP, Kilander MB, et al: Heterotrimeric $\mathrm{G}$ protein-dependent WNT-5A signaling to ERK1/2 mediates distinct aspects of microglia proinflammatory transformation. J Neuroinflammation 9: 111, 2012.

9. Kim J, Kim J, Kim DW, Ha Y, et al: Wnt5a induces endothelial inflammation via beta-catenin-independent signaling. J Immunol 185: 1274-1282, 2010.

10. Jung YS, Lee HY, Kim SD, et al: Wnt5a stimulates chemotactic migration and chemokine production in human neutrophils. Exp Mol Med 45: e27, 2013.

11. Malgor R, Bhatt PM, Connolly BA, et al: Wnt5a, TLR2 and TLR4 are elevated in advanced human atherosclerotic lesions. Inflamm Res 63: 277-285, 2014.

12. Yan X, Baxter RC, Perbal B and Firth SM: The aminoterminal insulin-like growth factor (IGF) binding domain of IGF binding protein-3 cannot be functionally substituted by the structurally homologous domain of CCN3. Endocrinology 147: 5268-5274, 2006.

13. Libby P, Ridker PM and Hansson GK: Inflammation in atherosclerosis: from pathophysiology to practice. J Am Coll Cardiol 54: 2129-2138, 2009.

14. Kutuk $\mathrm{O}$ and Basaga $\mathrm{H}$ : Inflammation meets oxidation: NF-kappaB as a mediator of initial lesion development in atherosclerosis. Trends Mol Med 9: 549-557, 2003.

15. Chan ED and Riches DW: IFN-gamma + LPS induction of iNOS is modulated by ERK, JNK/SAPK, and p38(mapk) in a mouse macrophage cell line. Am J Physiol Cell Physiol 280: C441-C450, 2001.

16. Schönbeck U, Sukhova GK, Graber P, Coulter S and Libby P: Augmented expression of cyclooxygenase- 2 in human atherosclerotic lesions. Am J Pathol 155: 1281-1291, 1999.

17. Burleigh ME, Babaev VR, Oates JA, et al: Cyclooxygenase-2 promotes early atherosclerotic lesion formation in LDL receptordeficient mice. Circulation 105: 1816-1823, 2002.

18. Burleigh ME, Babaev VR, Yancey PG, et al: Cyclooxygenase-2 promotes early atherosclerotic lesion formation in ApoE-deficient and C57BL/6 mice. J Mol Cell Cardiol 39: 443-452, 2005.

19. Gosling J, Slaymaker S, Gu L, et al: MCP-1 deficiency reduces susceptibility to atherosclerosis in mice that overexpress human apolipoprotein B. J Clin Invest 103: 773-778, 1999.

20. Falk E: Pathogenesis of atherosclerosis. J Am Coll Cardiol 47 (Suppl 8): C7-C12, 2006.

21. Davies MJ: The pathophysiology of acute coronary syndromes. Heart 83: 361-366, 2000.

22. Schaar JA, Muller JE, Falk E, et al: Terminology for high-risk and vulnerable coronary artery plaques. Report of a meeting on the vulnerable plaque, June 17 and 18, 2003, Santorini, Greece. Eur Heart J 25: 1077-1082, 2004.

23. Ylä-Herttuala S, Bentzon JF, Daemen M, et al: Stabilisation of atherosclerotic plaques. Position paper of the European Society of Cardiology (ESC) Working Group on atherosclerosis and vascular biology. Thromb Haemost 106: 1-19, 2011.

24. Naghavi M, Libby P, Falk E, et al: From vulnerable plaque to vulnerable patient: a call for new definitions and risk assessment strategies: Part I. Circulation 108: 1664-1672, 2003.

25. Newby AC: Metalloproteinase expression in monocytes and macrophages and its relationship to atherosclerotic plaque instability. Arterioscler Thromb Vasc Biol 28: 2108-2114, 2008.

26. Bond M, Fabunmi RP, Baker AH and Newby AC: Synergistic upregulation of metalloproteinase- 9 by growth factors and inflammatory cytokines: an absolute requirement for transcription factor NF-kappa B. FEBS Lett 435: 29-34, 1998.

27. Wang J, Zhang R, Xu Y, et al: Genistein inhibits the development of atherosclerosis via inhibiting NF-kappaB and VCAM-1 expression in LDLR knockout mice. Can J Physiol Pharmacol 86: 777-784, 2008

28. Muslin AJ: MAPK signalling in cardiovascular health and disease: molecular mechanisms and therapeutic targets. Clin Sci (Lond) 115: 203-218, 2008. 